(c) 2020 Universidad Nacional Autónoma de México, Facultad de Estudios Superiores Zaragoza.

Este es un artículo Open Access bajo la licencia CC BY-NC-ND (http://creativecommons.org/licenses/by-nc-nd/4.0/).

TIP Revista Especializada en Ciencias Químico-Biológicas, 23: 1-11, 2020.

DOI: $10.22201 /$ fesz.23958723e.2020.0.202

\title{
La batalla contra las superbacterias: No más antimicrobianos, no hay ESKAPE
}

\author{
Víctor M. Chávez-Jacobo \\ Instituto de Investigaciones Químico-Biológicas, Universidad Michoacana de \\ San Nicolás de Hidalgo, Ciudad Universitaria, Av. Gral. Francisco J. Mujica \\ s/n, Morelia, 58030, Michoacán. E-mail: victor_mch@hotmail.com
}

\begin{abstract}
RESUMEN
La resistencia a los antimicrobianos es uno de los más grandes retos de la medicina moderna. Durante la última década, un grupo de seis bacterias han probado no sólo su capacidad para relativamente "escapar" de los efectos de casi cualquier antimicrobiano, sino también por ser la causa principal de las infecciones hospitalarias. Estos organismos en conjunto se les conoce como ESKAPE, siglas que derivan de la primera letra de la categoría taxonómica género, o sea, del nombre científico de cada una de estas bacterias (Enterococcus spp, Staphylococcus aureus, Klebsiella pneumoniae, Acinetobacter baumannii, Pseudomonas aeruginosa y Enterobacter spp.). La presente revisión tiene como objetivo describir los principales mecanismos de resistencia asociados a este grupo de bacterias y el impacto que han tenido en el desarrollo de nuevas estrategias antimicrobianas.

Palabras Clave: Bacterias ESKAPE, infecciones hospitalarias, resistencia intrínseca, resistencia adquirida, selección de la resistencia.
\end{abstract}

The battle against the superbugs: No more antimicrobials, there is no ESKAPE

\begin{abstract}
Antimicrobial resistance is one of the greatest challenges of modern medicine. During the last decade, a group of six bacteria have proven not only their ability to relatively "escape" the effects of almost any antimicrobial but also because they are the main cause of hospital infections. These organisms together are known as ESKAPE, acronyms derived from the first letter of the genus taxonomic from the scientific name of each bacteria (Enterococcus spp, Staphylococcus aureus, Klebsiella pneumoniae, Acinetobacter baumannii, Pseudomonas aeruginosa y Enterobacter spp.) This review aims to describe the main resistance mechanisms associated with this group of bacteria and the impact they have had on the development of new antimicrobial strategies.
\end{abstract}

Key words: ESKAPE bacteria, hospital infections, intrinsic resistance, acquired resistance, selection of resistance. 


\section{INTRODUCCIÓN}

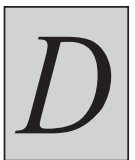
esde el descubrimiento de los antimicrobianos a principios del siglo XX, la medicina ha evolucionado en múltiples aspectos, salvando vidas gracias al uso de alguno de estos fármacos y convirtiéndose no sólo en uno de los grandes descubrimientos de la medicina moderna, sino también en medicamentos necesarios para el desarrollo de procedimientos médicos complejos como la amputación quirúrgica, el trasplante de órganos y el manejo de pacientes con cáncer, entre muchos otros (Munita \& Arias, 2016). Sin importar su estructura o función, que se mencionará más adelante, los antimicrobianos representan un grupo altamente diverso de moléculas que pueden inhibir el crecimiento (efecto bacteriostático) o provocar la muerte (efecto bactericida), específicamente de bacterias y han sido empleados durante décadas para controlar las infecciones en humanos, animales y plantas (Duijkeren, Schink, Roberts, Wang \& Schwarz., 2018).

Aunque el primer antimicrobiano que se produjo industrialmente fue la arsfenamina, un compuesto sintético empleado para tratar la sífilis (Aminov, 2017), hoy en día la mayoría de estos fármacos son productos naturales o derivados de los mismos, colectados a partir de microorganismos aislados del suelo, particularmente del género Streptomyces (Wright, Seiple \& Myers, 2014). Debido a su origen como productos derivados del metabolismo secundario bacteriano, muchos antimicrobianos se encuentran en la naturaleza, inclusive en la toma previa de muestras a la era del uso masivo de éstos (D'Costa et al., 2011) y como resultado de la ubicuidad en su producción ambiental, es posible encontrar bacterias que han desarrollado de manera ancestral mecanismos de defensa y por lo tanto de resistencia contra estos medicamentos (Crofts, Gasparrini \& Dantas, 2017). En los primeros días de la terapia antimicrobiana, la resistencia no era considerada un problema grave, debido a que el número de bacterias resistentes era muy bajo y se estaban detectando un gran número de antimicrobianos de diferentes clases (Tabla I). Desafortunadamente, la presión selectiva impuesta por el amplio uso y abuso de estos fármacos desde 1950 hasta nuestros días, ha acelerado el desarrollo y distribución de bacterias resistentes.

Hoy en día, las infecciones causadas por bacterias resistentes a múltiples antimicrobianos, que llamaremos superbacterias, son además un problema económico muy importante. Se estima que el gobierno de los Estados Unidos gasta alrededor de 20 mil millones de dólares al año en la prevención y tratamiento de enfermedades provocadas por estos microorganismos. Además, se considera que para 2050 las muertes prematuras causadas por bacterias multirresistentes alcanzarán cifras de 10 millones al año, mientras que actualmente es de 700,000 (O'Neil, 2016), por lo que la Organización Mundial de la Salud ha decretado a la resistencia antimicrobiana como uno de los principales problemas del siglo XXI (Tacconelli et al., 2018). Se pone especial atención en un grupo de bacterias denominadas ESKAPE (Enterococcus spp, Staphylococcus aureus, Klebsiella pneumoniae, Acinetobacter baumanni, Pseudomonas aeruginosa y Enterobacter spp.) (Rice, 2008) que serán descritas a través de la presente revisión con el objetivo de entender los mecanismos de resistencia asociados a este grupo de bacterias y el impacto que han tenido en el desarrollo de nuevas estrategias para combatir la resistencia.

\section{RESISTENCIA BACTERIANA}

La resistencia bacteriana puede dividirse de manera general en dos grupos: intrínseca y adquirida (Blair, Webber, Baylay, Ogbolu \& Piddock, 2014). Para comenzar a entender el problema de la resistencia, es necesario comprender que las bacterias han interactuado con los antimicrobianos de manera ancestral y por lo tanto han desarrollado mecanismos para resistir su efecto y sobrevivir o incluso prosperar en estos ambientes. Es por ello que estas bacterias se consideran intrínsecamente resistentes, no obstante, este grupo no es el principal foco del problema (Munita \& Arias, 2016). Por otro lado, la resistencia adquirida se da cuando una población bacteriana que era originalmente sensible a algún antimicrobiano se vuelve resistente mediante la adquisición de mutaciones en genes cruciales o mediante la adquisición externa de determinantes de resistencia transferidos de organismos intrínsecamente resistentes (Figura 1) (Blair et al., 2014). Debido a que no existen límites en la cantidad de determinantes que las bacterias pueden adquirir, han surgido poblaciones bacterianas altamente resistentes a múltiples familias de antimicrobianos, que denominamos como superbacterias.

La resistencia puede ser mediada por múltiples mecanismos que se pueden englobar dentro de tres tipos principales: Primero, aquellos que reducen la concentración intracelular del fármaco, ya sea impidiendo la entrada o a través de la expulsión; Segundo, la protección del sitio blanco mediante la adquisición de mutaciones en los genes que codifican para estos determinantes o mediante moléculas que interfieren con la unión del antimicrobiano; Tercero, la modificación del fármaco mediante hidrólisis o la adición de grupos funcionales (Figura 2) (Crofts et al., 2017).

\section{DisMinUCIÓN DE LA CONCENTRACIÓN DEL ANTIMICROBIANO}

Reducción de la permeabilidad

De manera general, las bacterias Gram (+) son menos permeables que las bacterias Gram (-) y por lo tanto sus membranas externas forman una barrera impermeable a muchos antimicrobianos (Kojima \& Nikaido, 2013). Los fármacos hidrofílicos pueden atravesar la membrana mediante difusión a través de proteínas de membrana llamadas porinas y las principales en Escherichia coli son OmpF y OmpC, 
Tabla I. Agentes antimicrobianos.

\begin{tabular}{|c|c|c|c|}
\hline Clase & Agente antimicrobiano & Organismo productor & $\begin{array}{c}\text { Año de aislamiento/ } \\
\text { descripción }\end{array}$ \\
\hline Arsfenaminas & Salvarsan & Sintético & 1907 \\
\hline B-Lactámicos & $\begin{array}{l}\text { Penicilinas } \\
\text { Cefalosporinas } \\
\text { Imipenem } \\
\text { Aztreonam }\end{array}$ & $\begin{array}{l}\text { Penicillum notatum } \\
\text { Cephalosporuim acremonium } \\
\text { Streptomyces cattleya } \\
\text { Chromobacterium violaceum }\end{array}$ & $\begin{array}{c}1929-1940 \\
1945-1953 \\
1976 \\
1981\end{array}$ \\
\hline Glucopéptidos & $\begin{array}{l}\text { Vancomicina } \\
\text { Teicoplanina, ovaparcina }\end{array}$ & $\begin{array}{l}\text { Amycolaptosis orientalis } \\
\text { Amycolaptosis coloradensis }\end{array}$ & $\begin{array}{c}\text { década de los } 50 \\
1975 \\
\end{array}$ \\
\hline Macrólidos & $\begin{array}{l}\text { Eritromicina } \\
\text { Espiramicina }\end{array}$ & $\begin{array}{l}\text { Streptomyces erythreus } \\
\text { Streptomyces ambofaciens }\end{array}$ & $\begin{array}{l}1952 \\
1955\end{array}$ \\
\hline Lincosamidas & Lincomicina & Streptomyces lincolnesis & 1963 \\
\hline Estreptograminas & $\begin{array}{l}\text { Estreptogramina } \mathrm{A}+\mathrm{B} \\
\text { Virginiamicina } \mathrm{A}+\mathrm{B}\end{array}$ & $\begin{array}{l}\text { Streptomyces diastaticus } \\
\text { Streptomyces virginiae }\end{array}$ & $\begin{array}{l}1953 \\
1955\end{array}$ \\
\hline Tetraciclinas & $\begin{array}{l}\text { Clortetraciclina } \\
\text { Oxitetraciclina }\end{array}$ & $\begin{array}{l}\text { Streptomyces aureofaciens } \\
\text { Streptomyces rimosus }\end{array}$ & $\begin{array}{l}1948 \\
1950\end{array}$ \\
\hline Fenicoles & Cloranfenicol & Streptomyces venezueleae & 1947 \\
\hline Aminoglucósidos & $\begin{array}{l}\text { Estreptomicina } \\
\text { Neomicina } \\
\text { Kanamicina } \\
\text { Gentamicina } \\
\text { Tobramicina } \\
\end{array}$ & $\begin{array}{l}\text { Streptomyces griseus } \\
\text { Streptomyces fradiae } \\
\text { Streptomyces kanamyceticus } \\
\text { Micromonospora purpura } \\
\text { Streptomyces tenebrarius } \\
\end{array}$ & $\begin{array}{l}1943 \\
1949 \\
1957 \\
1963 \\
1967 \\
\end{array}$ \\
\hline Aminociclitoles & Espectinomicina & Streptomyces spectabilis & 1961 \\
\hline Pleuromutilinas & Pleuromutilina, Tiamulina & Pleurotus sp., Sintético & 1951,1976 \\
\hline Polipéptidos & $\begin{array}{l}\text { Polimixina B } \\
\text { Colistina } \\
\text { Bacitracina } \\
\end{array}$ & $\begin{array}{l}\text { Bacillus polymyxa } \\
\text { B. Polymyxa var. colistinus } \\
\text { Bacillus licheniformis }\end{array}$ & $\begin{array}{l}1947 \\
1949 \\
1943 \\
\end{array}$ \\
\hline Epóxidos & Fosfomicina & Streptomyces fradiae & 1969 \\
\hline Ácidos pseudomónicos & Mupirocina & Pseudomonas fluorescens & 1971 \\
\hline Esteroides & Ácido fusídico & Fusidium coccineum & 1960 \\
\hline Estreptotricina & Nourseotricina & Streptomyces noursei & 1963 \\
\hline Sulfonamida & Sulfametoxazol & Sintético & 1935 \\
\hline Trimetoprima & Trimetoprim & Sintético & 1956 \\
\hline Quinolonas & Ácido Nalidíxico & Sintético & 1962 \\
\hline Fluoroquinolonas & Ciprofloxacina & Sintético & 1973 \\
\hline Oxazolidinonas & Linezolida & Sintético & 1987 \\
\hline
\end{tabular}


que actúan de manera inespecífica, al transportar desde nutrientes hasta colorantes, detergentes y antimicrobianos (Tran, Williams Farid, Erdemli \& Pearlstein, 2013). Debido a que reducir la permeabilidad de la membrana externa limita la entrada del antimicrobiano a la célula, las bacterias pueden disminuir la expresión de los genes que codifican para estas porinas y expresar genes que codifican para otras porinas. En Pseudomonas aeruginosa, Klebsiella pneumoniae y Acinetobacter baumannii la reducción en la expresión de porinas contribuye de manera significativa con la resistencia a B-lactámicos (Pang, Raudonis, Glick, Lin \& Cheng, 2019; Baroud et al., 2013; Lavigne et al, 2013).

\section{Expulsión del antimicrobiano}

Los sistemas de expulsión bacterianos transportan de manera activa a la gran mayoría de los antimicrobianos mencionados en la Tabla I y son los principales responsables de la resistencia intrínseca. Mientras que algunos sistemas de transporte tienen sustratos específicos, por ejemplo, los sistemas Tet, que expulsan de manera específica tetraciclinas, muchos otros pueden expulsar una gran cantidad de sustancias con estructuras totalmente distintas y a éstos se les conoce como sistemas de expulsión múltiple (Du et al., 2018). Actualmente, se han identificado seis familias diferentes de transportadores bacterianos capaces de conferir resistencia a los antimicrobianos, uno de ellos son los de unión al ATP (ABC por sus siglas en inglés, ATP-binding cassette) que utilizan directamente al ATP como fuente de energía para realizar el transporte, mientras que las otras cinco familias de transportadores funcionan a través del potencial de membrana y son: facilitadores mayores (MFS, major facilitat or superfamily), expulsión de antimicrobianos y toxinas (MATE, multidrug and toxin extrusion), proteínas pequeñas de resistencia a antimicrobianos (SMR, small multidrug resistance), resistencia, nodulación $\mathrm{y}$ división celular (RND, resistance-nodulation-cell division) y los sistemas de expulsión de proteobacterias (PACE, proteobacterial, antimicrobial compound efflux) (Du et al., 2018).

Todas las bacterias contienen diversos genes que codifican sistemas de expulsión múltiples dentro de sus cromosomas, algunos pueden ser movilizados a través de plásmidos,

\section{a) Evolución vertical}

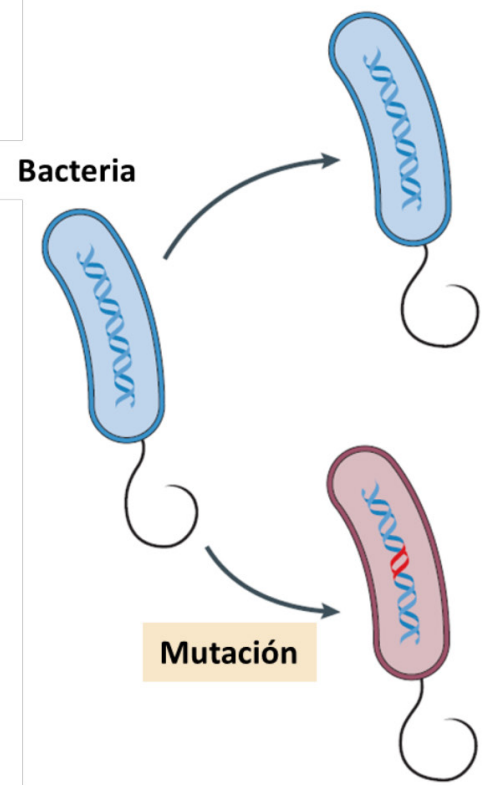

b) Evolución horizontal

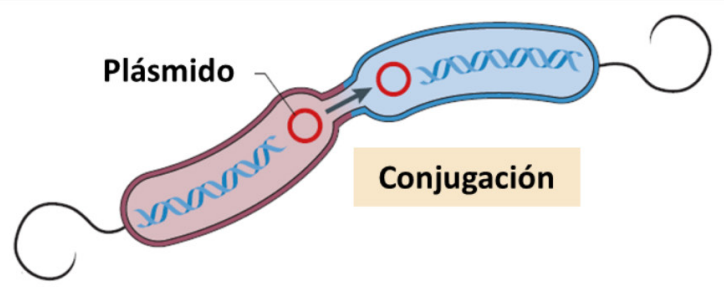

Transformación

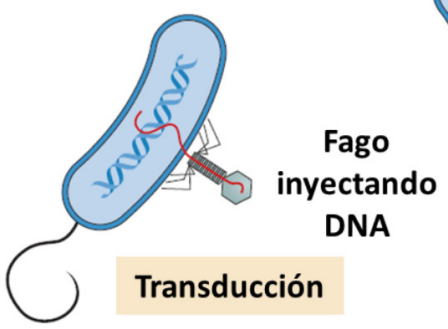

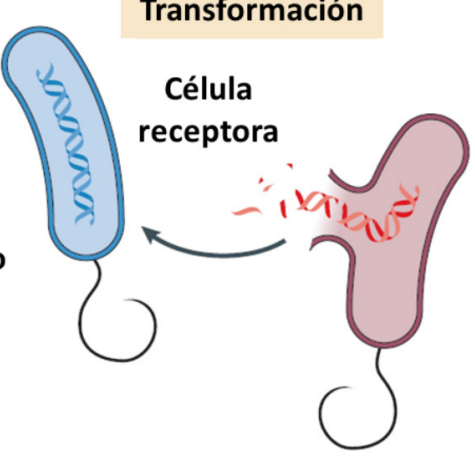

Figura 1. Evolución de la resistencia. La resistencia puede evolucionar a través de dos mecanismos básicos. Evolución vertical y horizontal. a) La evolución vertical representa la generación de una nueva mutación en el genoma bacteriano y la subsecuente transferencia a su progenie. Las bacterias azules son sensibles a antimicrobianos, mientras que, las rojas son resistentes. b) La adquisición de genes de resistencia de manera horizontal puede ocurrir mediante transducción, conjugación o transformación. La transducción es el proceso mediante el cual un bacteriófago introduce material genético al interior de una célula bacteriana y éste se incorpora a su genoma. La conjugación ocurre mediante el contacto directo entre dos células bacterianas, una donadora y la otra receptora y media la transferencia de plásmidos conjugativos y mobilizables así como de elementos integrativos. La transformación ocurre cuando el material genético se libera mediante la lisis de la célula donadora y la célula receptora lo incorpora a su genoma. Todos estos procesos median la adquisición de genes que confieren resistencia. Las células azules son receptoras, mientras que las rojas son donadoras (Modificado de Sommer, Munck, Toft-Kehler \& Andersson, 2017). 


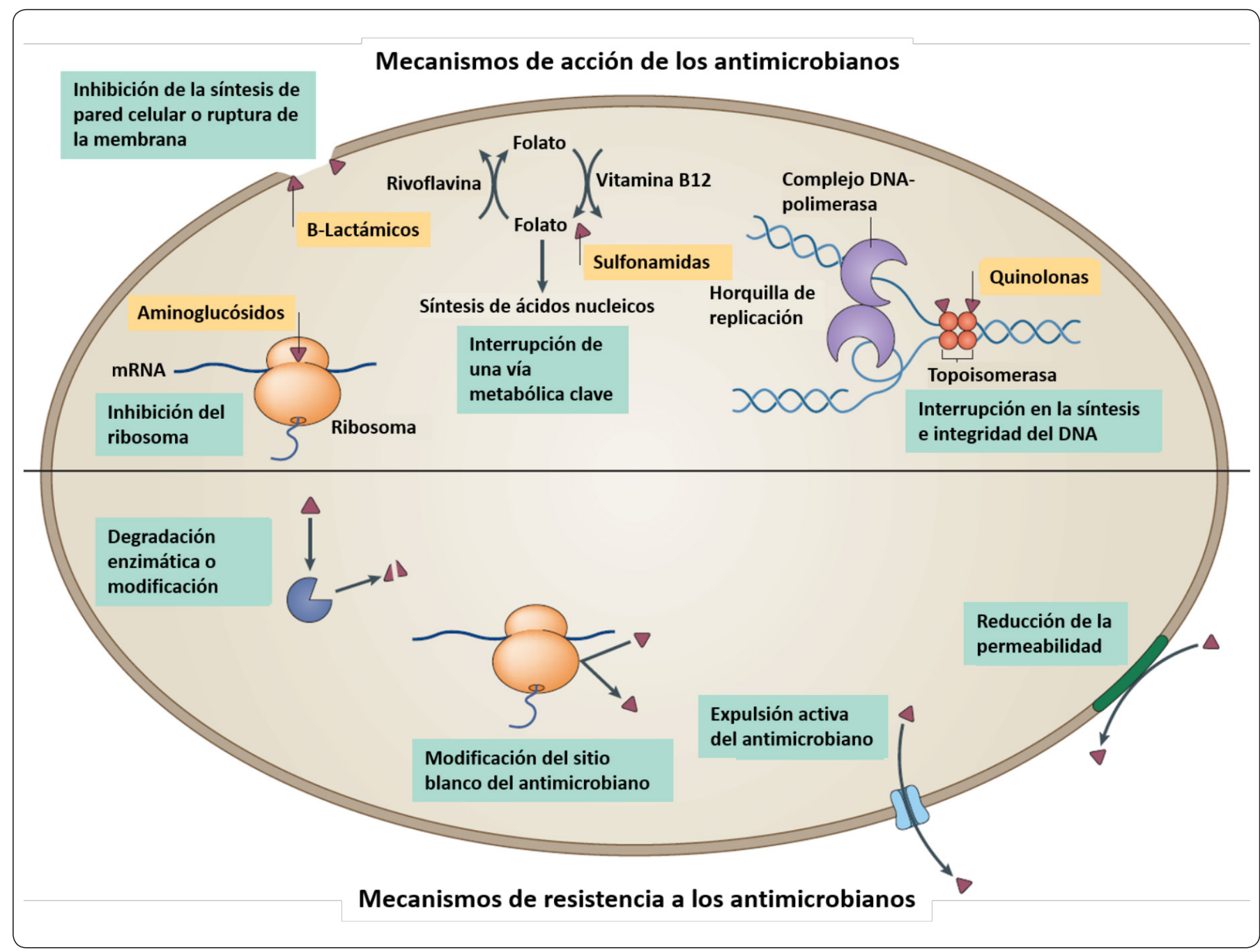

Figura 2. Mecanismos de resistencia a los antimicrobianos. Los blancos de los antimicrobianos se encuentran conservados entre las bacterias y normalmente se encuentran ausentes o son los suficientemente diferentes en células eucariotas. Aunque existan cientos de blancos potenciales, el arsenal de antimicrobianos con el que contamos ataca principalmente al ribosoma, la síntesis de pared celular, la interrupción de una vía metabólica clave y el metabolismo del DNA. Las células bacterianas comúnmente resisten la acción de los antimicrobianos mediante la inactivación del antimicrobiano a través de enzimas que degradan o modifican al fármaco. Otras estrategias de resistencia son la protección, alteración o sobreexpresión del blanco y una última estrategia consiste en la expulsión del antimicrobiano a través de sistemas de expulsión tanto específicos como inespecíficos (Modificado de Crofts et al., 2017).

transposones o bacteriófagos (Figura 1) por lo que estos sistemas están ampliamente distribuidos y en la actualidad aproximadamente el $80 \%$ de las infecciones hospitalarias severas se atribuyen a bacterias Gram (-) con sistemas de expulsión múltiple activos (Viale, Giannella, Tedeschi \& Lewis, 2015). Se conoce, además, que para que estos sistemas sean aún más efectivos pueden sobreexpresarse, es decir, aumentar el número de proteínas transportadoras presentes en las células y por lo tanto aumentar aún más la expulsión. Desde 1990, se han aislado cepas de P. aeruginosa y $S$. aureus capaces de sobreexpresar sistemas de expulsión (Pumbwe \& Piddock, 2000; Kosmidis et al., 2012), por lo que el entendimiento de estos sistemas es importante debido a que es muy común encontrarlo en superbacterias y hoy en día es urgente diseñar nuevas estrategias para prevenir la expulsión de los antimicrobianos.

\section{Protección del Sitio Blanco Del ANTI- MICROBIANO}

Mutación de genes que codifican el sitio blanco

La mayoría de los antimicrobianos tienen alta afinidad por su sitio blanco y una vez que se unen con éste, tienen la capacidad de desestabilizar las funciones normales de un organelo específico o una vía metabólica fundamental para la célula bacteriana, por lo que cambios en la estructura del blanco que impidan la unión del fármaco pero que permitan 
continuar con su funcionamiento normal, confieren resistencia a los antimicrobianos (Figura 2). Durante el transcurso de una infección bacteriana puede ocurrir un fenómeno llamado selección, donde dentro de una población de bacterias surgen algunas que pueden desarrollar una mutación simple que confiere resistencia y por lo tanto pueden proliferar, a pesar del antimicrobiano y volver obsoleta la terapia contra ellas. Para el caso de las superbacterias se reduce significativamente el número de fármacos viables, ya que pueden acumular múltiples mutaciones en diversos genes y sobrevivir a una gran cantidad de antimicrobianos.

\section{Interferencia con la unión del antimicrobiano}

La protección del sitio blanco es una manera muy efectiva de protegerse contra el efecto de un antimicrobiano y básicamente consiste en sintetizar una molécula que pueda modificar al blanco sin inhibirlo o pueda unirse al antimicrobiano y por lo tanto bloquearlo. En los últimos años la protección de los sitios blanco se ha vuelto de interés clínico debido a la resistencia conferida contra importantes antimicrobianos: por ejemplo, la protección mediada por la metilasa del RNA ribosomal 16S, codificada por el gen erm, que previene la unión de macrólidos, lincosamidas y estreptograminas (Shore et al., 2011). Es decir, sólo un factor de resistencia puede inactivar a tres diferentes familias de antimicrobianos. Otro ejemplo de interferencia son las proteínas Qnr que con capaces de bloquear la unión de las quinolonas con su sitio blanco, las topoisomerasas II y IV, y de esta manera conferir resistencia (Goto, Kawamura \& Arakawa, 2015).

\section{MODIFICACIÓN DE LOS ANTIMICROBIANOS Modificación mediante hidrólisis}

La modificación enzimática de los antimicrobianos es uno de los mecanismos más eficientes para inactivarlos y ha sido un problema desde la introducción de los B-lactámicos en 1940. Actualmente han sido reportadas cientos de enzimas capaces de degradar o modificar B-lactámicos, aminoglucósidos, fenicoles y macrólidos. El aumento en el número de antimicrobianos de la misma familia a los cuales se les han introducido modificaciones para hacerlos más efectivos, ha provocado que las enzimas capaces de degradarlos incrementen su espectro de acción y hoy en día, tenemos B-lactamasas de espectro extendido (ESBLs), enzimas capaces de romper B-lactámicos, que proporcionan resistencia a todos los antimicrobianos de esta familia, y que se encuentran comúnmente en aislados de $K$. pneumoniae, E. coli, $P$. aeruginosa y A. baumannii (Rossolini, D'Andrea \& Mugnaioli, 2008). En algunos casos el rápido aumento en la diseminación de bacterias resistentes a B-lactámicos se ha tratado de explicar con la capacidad de estos determinantes de transferirse a través de plásmidos. Sea cual sea la explicación, el fenómeno de resistencia a B-lactámicos se ha expandido muy rápido $\mathrm{y}$ hoy es uno de los principales retos en el desarrollo de nuevas estrategias antimicrobianas.

\section{Modificación mediante la adición de grupos funcionales}

La adición enzimática de grupos funcionales provoca resistencia debido a que disminuye la afinidad del antimicrobiano por su sitio blanco y las enzimas mejor estudiadas capaces de transferir estos grupos funcionales son las aminoglucósido transferasas (Smith \& Baker. 2002).

Los aminoglucósidos son particularmente susceptibles a la adición de grupos funcionales, debido a que su estructura cuenta con diversos grupos hidroxilo y amino expuestos. Existen tres clases diferentes de aminoglucósidos transferasas: las acil transferasas, fosfo transferasas y nucleotidil transferasas las cuales confieren altos niveles de resistencia y se encuentran ampliamente distribuidas (Serio, Keepers, Andrews \& Krause, 2018). Además, se han reportado dos enzimas pertenecientes a las aminoglucósido transferasas que son capaces de modificar a la ciprofloxacina (un antimicrobiano sintético de la familia de las quinolonas), la AAC-Ib-cr, que es capaz de adicionar un grupo acilo (Robicsek et al., 2006) y CrpP, que es capaz de adicionar un grupo fosfato (Chávez-Jacobo et al., 2018). Por lo que la visión hacia el futuro es muy preocupante, ya que la resistencia, que este grupo de enzimas proporcionaba y que parecía ser exclusiva para aminoglucósidos, se ha extendido hacia un grupo de antimicrobianos completamente diferente. Además, se ha reportado que $\mathrm{CrpP}$, descubierto inicialmente en un plásmido de $P$. aeruginosa, se encuentra distribuido en E. coli y en K. pneumoniae (Chávez-Jacobo et al., 2019).

\section{Superbacterias ESKAPE}

El término ESKAPE hace referencia a un grupo de seis bacterias responsables de la mayoría de las infecciones nosocomiales que son capaces de, relativamente, escapar de los efectos de los antimicrobianos. Dentro de este grupo se incluyen Enterococcus spp, Staphylococcus aureus, Klebsiella pneumoniae, Acinetobacter baumannii, $\boldsymbol{P}$ seudomonas y aeruginosa Enterobacter spp. (Rice, 2008). Debido a la amplia distribución de infecciones causadas por este grupo de bacterias el Centro para el Control de Enfermedades de los Estados Unidos (CDC) ha emitido una alerta para incentivar la creación de nuevas estrategias para el tratamiento de infecciones causadas principalmente por las siguientes bacterias: $S$. aureus resistente a meticilina (MRSA), E. feacium resistente a vancomicina, $P$. aeruginosa resistente a fluoroquinolonas, Enterobacteriaceas productoras de B-lactamasas de espectro extendido (ESBL) y Acinetobacter baumannii resistente a carbapenémicos (Nakonieczna et al., 2019).

Enterococcus spp. es un género de bacterias Gram $(+)$, anaerobias facultativas, catalasa negativas y no esporuladas, que se pueden encontrar distribuidas extensamente en la naturaleza, tanto en suelo, agua y plantas, pero también formar parte de la flora normal del tracto entérico de humanos y animales (Fisher \& Phillips, 2009). Enterococcus feacium 
y Enterococcus feacalis son los principales patógenos de este grupo, asociados con una gran variedad de enfermedades como: infecciones de heridas, bacteremia, infecciones de vías urinarias, infecciones dentales, endocarditis, infecciones abdominales y sepsis neonatal (Fisher \& Phillips, 2009). Además, en Europa se han aislado Enterococcus resistentes a vancomicina, en carne (Messi, Guerrieri, Niederhaussern, Sabia \& Bondi, 2006) y en productos lácteos (Ogier \& Serror. 2008), lo cual convierte a estos alimentos contaminados en un gran riesgo sanitario.

Tradicionalmente, para las infecciones causadas por $E$. feacium se empleaban B-lactámicos o glucopéptidos; sin embargo, la resistencia para los primeros es casi total, por ejemplo, para ampicilina e imipenem tenía una prevalencia del 98.8\% en Reino Unido en el 2006 (Brown et al., 2008) y hoy en día se espera que la resistencia sea total, por lo que este grupo de antimicrobianos es completamente inútil en el tratamiento de infecciones causadas por esta bacteria. Para el caso de los glucopéptidos, específicamente vancomicina, existen al menos nueve determinantes de resistencia, vanA, $\mathrm{B}, \mathrm{C}, \mathrm{D}, \mathrm{E}, \mathrm{G}, \mathrm{L}, \mathrm{M}$ y $\operatorname{van} \mathrm{N}$, siendo vanA el más distribuido alrededor del mundo y el más comúnmente asociado con la resistencia de E. feacium (Kang et al., 2014).

Staphylococcus aureus es una bacteria Gram (+) con un crecimiento característico formando lo que se denomina "racimos de uvas". Forma parte de la flora normal de la piel y se encuentra principalmente en el tracto nasal y en el perineo de los mamíferos (Appelbaum, 2007). S. aureus cuenta con todo un arsenal de proteínas extracelulares que emplea durante la infección como factores de virulencia: hemolisinas, proteasas, hialuronidasas y colagenasas que le ayudan a la colonización y en la búsqueda de nutrientes (Boubaker et al., 2004). Normalmente, las infecciones causadas por $S$. aureus eran tratadas con penicilina, sin embargo, el uso y abuso de este tratamiento ha causado que la resistencia se extienda, por lo que actualmente el $90 \%$ de los aislamientos clínicos de $S$. aureus son resistentes a la penicilina y aproximadamente el $60 \%$ de éstos son resistentes a la meticilina (MRSA), que era empleado como última opción (Sakoulas \& Moellering, 2008).

El tratamiento de primera línea para las infecciones causadas por bacterias MRSA es la vancomicina, sin embargo, una vez más, la fuerte presión selectiva causada por el extenso uso de este antimicrobiano ha causado la aparición de dos tipos de resistencia, intermedia y completa. S. aureus, con resistencia intermedia, ha evolucionado para generar una membrana poco permeable a la mayoría de los antimicrobianos, por lo que es virtualmente insensible a la mayoría de los tratamientos empleados, lo cual, sumado con los factores de virulencia, la convierte en una superbacteria que pone en riesgo a todas las personas, especialmente a las que tienen problemas inmunitarios (Vestergaard, Frees \& Ingmer, 2019). El primer reporte de resistencia intermedia fue emitido en Japón a mediados de los años 90 y se ha vuelto un problema de interés global, debido a la cada vez más frecuente aparición de cepas con resistencia intermedia en Asia, Europa y América (Chambers \& DeLeo, 2009). La resistencia completa es muy poco común y ha surgido debido a la transferencia de genes inter-especies con Enterococcus resistentes a la vancomicina, ya que se han identificado $S$. aureus con genes vanA (Gardete \& Tomasz, 2014).

Klebsiella pneumoniae es un miembro de la familia Enterobacteriaceae, donde también se agrupa Escherichia coli: es un bacilo Gram (-) y se considera una bacteria intrínsecamente virulenta debido a la presencia de adhesinas y de una cápsula que actúa como un factor antifagocítico (Clegg \& Murphy, 2016). Se considera un patógeno oportunista y se asocia comúnmente con infecciones de las vías urinarias y de las vías respiratorias. K. pneumoniae es notoria por la acumulación y rápida diseminación de múltiples determinantes de resistencia a los antimicrobianos: uno de los más importantes es la adquisición de una gran variedad de B-lactamasas, enzimas capaces de hidrolizar el anillo B-lactámico común en todas las penicilinas, cefalosporinas y carbapenémicos. Debido a que los carbapenémicos son los antimicrobianos de primera línea en el tratamiento de infecciones causadas por esta bacteria, las opciones de tratamiento son limitadas y en países como Estados Unidos, K. pneumoniae productora de carbapenemasas (KPC) ha sido el agente causal de infecciones a gran escala, debido principalmente a que el determinante de resistencia es capaz de transferirse entre diferentes especies y en Reino Unido es causante del $20 \%$ de las bacteriemias (Queenan \& Bush. 2007. Livermore, Hope, Brick, Lillie \& Reynolds, 2008).

El panorama completo es aún más complicado, ya que, en el laboratorio, cepas altamente virulentas de $K$. pneumoniae son capaces de formar un biofilm, una matriz polimérica compuesta por polisacáridos y proteínas que le confieren protección contra elementos exógenos del medio, como los antimicrobianos (Clegg \& Murphy, 2016). En la naturaleza, son capaces de asociarse con otras especies bacterianas que comparten una distribución espacial similar: por ejemplo, se han reportado asociaciones con $P$. aeruginosa, Proteus mirabilis y Candida albicans, entre otros. Mientras que las interacciones que se llevan a cabo en estas comunidades complejas permanecen sin resolver, por el momento sólo se ha podido establecer el hecho de que lidiar con infecciones de esta naturaleza es sumamente complicado (Nakonieczna et al., 2019).

Acinetobacter baumannii es un cocobacilo aeróbico, Gram (-), no presenta movilidad y no es capaz de fermentar glucosa. Se considera un patógeno oportunista y es el 
responsable del $2-10 \%$ de las infecciones hospitalarias causadas por bacterias Gram (-) y se asocia comúnmente con infecciones en la piel, bacteriemia, meningitis, neumonía e infección de heridas (Lee et al., 2017). A. baumannii puede ingresar fácilmente al cuerpo humano a través de heridas abiertas, catéteres intravasculares y ventiladores mecánicos, debido a su capacidad para crecer en un gran intervalo de temperaturas, $\mathrm{pH}$ y niveles de nutrientes, puede sobrevivir dentro del cuerpo humano, así como en diferentes vectores (Antunes, Visca \& Towner, 2014). Además, A. baumannii posee una citotoxina, que consiste en una proteína de membrana externa llamada Omp38, que es capaz de inducir la apoptosis de las células epiteliales humanas y se considera un factor de virulencia crucial para las primeras fases de la infección (Choi et al., 2005).

Un fenómeno observado desde hace algunos años ha sido la asociación de infecciones causadas por A. baumannii en heridas producidas en zonas de conflictos armados; por ejemplo, en veteranos y soldados que estuvieron en Irak y Afganistán se detectaron 85 casos, donde el 35\% de las cepas fueron susceptibles únicamente a una clase de antimicrobiano y el $4 \%$ fueron resistentes a todos los tratamientos estándar (Peleg, Seifert \& Paterson, 2008). Existen evidencias que indican que el efecto inmunosupresor de la morfina puede incrementar el riesgo de contraer una infección causada por esta bacteria: no obstante, es el tratamiento habitual después de sufrir una herida en combate (Breslow et al., 2011).

La principal preocupación en el caso de A. baumannii ha sido la rápida aparición y propagación de cepas que contienen B-lactamasas de espectro extendido, ya que éstas muestran resistencia a colistina e imipenem. Además, la combinación con otros factores de -resistencia intrínsecos, como son la presencia de sistemas de expulsión, ha provocado que esta bacteria sea capaz de evadir la acción de la gran mayoría de los tratamientos estándar que consistían anteriormente en el uso de carbapenémicos, B-lactámicos y fluoroquinolonas (Lee et al., 2017).

Pseudomonas aeruginosa es una bacteria Gram (-), anaerobia facultativa, que puede encontrarse en animales, plantas y suelo y ha sido reconocida como la bacteria patógena oportunista más asociada a infecciones hospitalarias en pacientes que utilizan ventiladores (Lupo, Haenni \& Madec, 2018). En personas sanas raramente puede llegar a afectar su salud, sin embargo, presenta una mortalidad alta en pacientes con fibrosis quística y en individuos inmunocomprometidos (Pang, Raudonis, Glick, Lin \& Cheng, 2019). Esta bacteria presenta una resistencia intrínseca a múltiples antimicrobianos: especialmente puede desarrollar resistencia durante el tratamiento con imipenem, que anteriormente era el tratamiento de elección, mediante la sobreexpresión o la modificación del gen que codifica a la porina AmpC (Pang et al., 2019). P. aeruginosa puede producir también B-lactamasas de espectro extendido y han surgido aislados capaces de resistir los efectos de las fluoroquinolonas, que llegaron a ser consideradas como la nueva esperanza en el tratamiento de infecciones causadas por esta bacteria, mediante la mutación de los genes que codifican los blancos de las fluoroquinolonas, la DNA girasa y la topoisomerasa IV, así como con el reciente reporte del determinante de resistencia $\mathrm{CrpP}$ codificado en un plásmido (Chávez-Jacobo et al., 2018).

Durante las primeras etapas de la colonización, $P$. aeruginosa se adhiere a las células gracias a la presencia de flagelos y de un pili, que es un apéndice especial de naturaleza proteínica que además se emplea en la transferencia de material genético entre especies. Una vez que la adhesión es exitosa, $P$. aeruginosa comienza a secretar dos colorantes, que utiliza como sideróforos, moléculas especializadas en la quelación del hierro y como resultado las células hospederas entran en estado de privación de hierro, lo cual activa una vía de respuesta inmune innata, que en personas sanas acabaría rápidamente con la infección, pero que en personas inmunocomprometidas su única opción es el uso de fármacos antimicrobianos (Nikaido, 2003).

Enterobacter spp. un bacilo Gram (-), anaerobio facultativo que se puede encontrar encapsulado y se asocia comúnmente con infecciones de las vías urinarias y del tracto respiratorio, aunque también se encuentra en infecciones sanguíneas en pacientes inmuno-comprometidos (Mezzatesta, Gona \& Stefani, 2012). Enterobacter cloacae y Enterobacter aerogenes son las bacterias más representativas de este grupo y las más comúnmente asociadas con multirresistencia a antimicrobianos. Aunque se han reportado aislados resistentes a B-lactámicos y fluoroquinolonas, que eran la primera opción para tratar este tipo de infecciones, hasta el momento el empleo de colistina ha dado buenos resultados (Davin-Regli, Lavigne \& Pagés, 2019).

Fue en 1989 cuando se reportó el primer caso de infecciones hospitalarias debido a $E$. cloacae productora de B-lactamasas de espectro extendido (De Champs et al., 1989). Actualmente, junto con E. coli y $K$. pneumoniae representan a las tres enterobacterias con mayor número de casos de resistencia a los antimicrobianos. La resistencia se encuentra ampliamente distribuida y para imipenem se reporta hasta el $0.4 \%$ (Lee et al., 2005), para aminoglucósidos hasta el 51\%, y para fluoroquinolonas del 64 al 100\% (Corkill, Anson \& Hart, 2005), por lo que las opciones para el correcto tratamiento de infecciones causadas por Enterobacter sp son limitadas.

\section{Conclusiones}

Actualmente, existe una urgente necesidad de desarrollar nuevas estrategias para combatir específicamente la resistencia a los antimicrobianos del grupo de bacterias 
ESKAPE, ya que este grupo es la mayor amenaza a los sistemas de salud pública en todo el mundo, por lo que es necesaria una rápida acción para tratar este problema tan grave. Hasta el momento no ha sido suficiente la implementación de terapias empleando combinaciones de fármacos y desafortunadamente, no parecen existir avances en el desarrollo de nuevos antimicrobianos contra $P$. aeruginosa y $A$. baumannii. Se han hecho grandes esfuerzos en el desarrollo de nuevos inhibidores de B-lactamasas y de sistemas de expulsión; sin embargo, esto no ha sido suficiente y por el momento es urgente explorar nuevas alternativas, como podrían ser: terapia con bacteriófagos acoplados con la tecnología CRISPR/Cas (Chávez-Jacobo, 2018) y el uso de terapia fotodinámica (Nakonieczna et al., 2019). Por el momento tenemos que poner todas nuestras esperanzas en el desarrollo y perfeccionamiento de estas nuevas estrategias, ya que siguen siendo inestables y resultan tóxicas para el paciente. Sin embargo, podrían convertirse en la mejor alternativa para tratar infecciones causadas principalmente por el grupo de bacterias ESKAPE.

\section{Agradecimientos}

Como alumno del Programa Institucional de Doctorado en Ciencias Biológicas: Opción Biología Experimental de la Universidad Michoacana de San Nicolás de Hidalgo agradezco la beca otorgada por el CONACYT (483024).

\section{REFERENCIAS}

Aminov, R. (2017). History of antimicrobial drug discovery - Major classes and health impact. Biochem. Pharmaco., 133, 4-19. https://doi.org/10.1016/j.bcp.2016.10.001.

Antunes, L. C. S., Visca, P. \& Towner, K. J. (2014). Acinetobacter baumannii: evolution of a global pathogen. Pathog. Dis., 71(3), 292-301. https://doi. org/10.1111/2049-632X.12125.

Appelbaum, P. C. (2007). Microbiology of antibiotic resistance in Staphylococcus aureus. Clin. Infect. Dis., 45(3), 165170. https://doi.org/10.1086/519474.

Baroud, M., Dandache, I., Araj, G. F., Wakim, R., Kanj, S., Kanafani, Z., Khairallah, M., Sabra, A., Shehab, M., Dbaibo, G. \& Matar, G. M. (2013). Underlaying mechanisms of carbapenem resistance in extendedspectrum $\beta$-latamase-producing Klebsiella pneumoniae and Escherichia coli isolates at a tertiary care centre in Lebanon: role of OXA-48 and NDM-1 carbapenemases. Int. J. Infect. Dis., 41, 75-79. https://doi.org/10.1016/j. ijantimicag.2012.08.010.

Boubaker, K., Diebold, P., Blanc, D. S., Vandenesch, F., Praz, G., Dupuis, G. \& Troillet, N. (2004). PantonValentine leukocidin and Staphyloccoccal skin infections in schoolchildren. Emerg. Infect. Dis., 10(1), 121-124. https://doi.org/10.3201/eid1001.030144.

Blair, J. M. A., Webber, M. A., Baylay, A. J., Ogbolu, D. O. \& Piddock, L. J. V. (2014). Molecular mechanisms of antibiotic resistance. Nat. Rev. Microbiol., 13(1), 42-51. https://doi.org/10.1038/nrmicro3380.

Breslow, J. M., Monroy, M. A., Daly, J. M., Meissler, J. J., Gaugham, J., Adler, M. A. \& Eisenstein, T. K. (2011). Morphine, but not trauma, sensitizes to systemic Acinetobacter baumannii infections. J. Neuroimmune Pharmacol., 6(4), 551-565. https://doi.org/10.1007/ s11481-011-9303-6.

Brown, D. F. J., Hope, R., Livermore, D. M., Brick, G., Broughton, K., George, R. C. \& Reynolds, R. (2008). Non-susceptibility trends among enterococci and nonpneumococcal streptococci from bacteraemias in the UK and Ireland, 2001-06. J. Antimicrob. Chemother 62(2), 75-85. https://doi.org/10.1093/jac/dkn354.

Chambers, H. F. \& DeLeo, F. R. (2009). Waves of resistance: Staphylococcus aureus in the antibiotic era. Nat. Rev. Microbiol., 7(9), 629-641. https://doi.org/10.1038/ nrmicro2200.

Chávez-Jacobo, V. M. (2018). El sistema de edición genética CRISPR/Cas y su uso como antimicrobiano especifico. TIP Rev. Esp. Cienc. Quím. Biol., 21(2), 116-123. https:// doi.org/10.22201/fesz.23958723e.2018.2.138.

Chávez-Jacobo, V. M., Hernández-Ramírez, K. C., RomoRodríguez, P., Pérez-Gallardo, R. V., Campos-García, J., Gutiérrez-Corona, J. F., García-Merinos, J. P., MezaCarmen, V., Silva-Sánchez, J. \& Ramírez-Díaz, M. I. (2018). CrpP is a novel ciprofloxacin-modifying enzyme encoded by the Pseudomonas aeruginosa pUM505 plasmid. Antimicrob. Agents Chemother, 62 (6), e0262917. https://doi.org/10.1128/AAC.02629-17.

Chávez-Jacobo, V. M., Hernández-Ramírez, K. C., SilvaSánchez, J., Garza-Ramos, U., Barrios Camacho, H., Ortiz-Alvarado, R., Meza-Carmen, V., Silva-Sánchez, J. \& Ramírez-Díaz, M. I. (2019). Prevalence of the crpP gene conferring decreased ciprofloxacin susceptibility in enterobacterial clinicali solates from Mexican Hospitals. J. Antimicrob. Chemother. DOI: 10.1093/jac/dky562 https://doi.org/10.1093/jac/dky562.

Choi, C. H., Lee, E. Y., Lee, Y. C., Park, T. I., Kim, H. J., Hyun, S. H., Kim, H. J., Hyun, S. H., Kim, S. A., Lee, S. \& Lee, J. C. (2005). Outer membrane protein 38 of Acinetobacter baumannii localizes to the mitochondria and induces apoptosis of epithelial cells. Cell Microbiol., 7(8), $1127-$ 1138. https://doi.org/10.1111/j.1462-5822.2005.00538.x.

Clegg, S. \& Murphy, C. N. (2016). Epidemiology and virulence of Klebsiella pneumoniae. Microbiol. Spctr., 4(1), UTI-0005. https://doi.org/10.1128/microbiolspec. UTI-0005-2012.

Crofts, T. S., Gasparrini, A. J. \& Dantas, G. (2017). Nextgeneration approaches to understand and combat the antibiotic resistome. Nat. Rev. Microbiol., 15(7), 422434. https://doi.org/10.1038/nrmicro.2017.28.

Corkill, J. E., Anson, J. J. \& Hart, A. (2005). High prevalence of plasmid-mediated quinolone resistance determinant qnrA 
in multidrug-resistant Enterobacteriaceae from blood cultures in Liverpool, UK. J. Antimicrob. Chemother, 56, 1115-1117. https://doi.org/10.1093/jac/dki388.

Davin-Regli, A., Lavigne, J. \& Pagés, J. (2019). Enterobacter spp.: Update on taxonomy, clinical aspects, and emerging antimicrobial resistance. Clin. Microbiol. Rev. 32(4), e00002-19. https://doi.org/10.1128/CMR.00002-19.

De Champs, C., Sauvant, M. P., Chanal, C., Sirot, D., Gazuy, N., Malhuret, R., Baguet, J. C. \& Sirot, J. (1989). Prospective survey of colonization and infection caused by expanded-spectrum- $\beta$-lactamases-producing members of the family Enterobacteriaceae in an intensive care unit. J. Clin. Microbiol., 27(12), 2887-2890.

Du, D., Wang-Kan., X., Neuberger, A., Veen, H. W., Pos, K. M., Piddock, L. J. V. \& Luisi, B. F. (2018). Multidrug efflux pumps: structure, function and regulation. Nat. Rev. Microbiol., 16(9), 523-239. https://doi.org/10.1038/ s41579-018-0048-6.

D'Costa, V. M., King, E. E., Kalan, L., Morar, M., Sung, W. W. L., Schwarz, C., Froese, D., Zazula, G., Calmels, F., Debruyne, R., Golding, G. B., Poinar, H. N. \& Wright, G. D. (2011). Antibiotic resistance is ancient. Nature, 477, 457-561. https://doi.org/10.1038/nature10388.

Duijkeren, E., Schink, A., Roberts, M. C., Wang, Y. \& Schwarz, S. (2018). Mechanisms of bacterial resistance to antimicrobial agents. Microbiol. Spectr. 6(1), ARBA0019. https://doi.org/10.1128/microbiolspec.ARBA0019-2017.

Fisher, K \& Phillips C. (2009). The ecology, epidemiology and virulence of Enterococcus. Microbiology, 155, 17491757. https://doi.org/10.1099/mic.0.026385-0.

Gardete, S. \& Tomasz, A. (2014). Mechanisms of vancomycin resistance in Staphylococcus aureus. J. Clin. Invest., 124(7), 2836-2840. https://doi.org/10.1172/JCI68834.

Goto, K., Kawamura, K. \& Arakawa, Y. (2015). Contribution of QnrA, a plasmid-mediated quinolone resistance peptide, to survival of Escherichia coli exposed to a lethal ciprofloxacin concentration. Jpn. J. Infect. Dis., 68, 196202. https://doi.org/10.7883/yoken.JJID.2014.153.

Kang, M., Xie, Y., He, C., Chen, Z. Y., Guo, L., Yang, Q., Liu, J. Y., Du, Y., Ou, Q. S. \& Wang, L. L. (2014). Molecular characteristics of vancomycin-resistant Enterococcus feacium from a tertiary care hospital in Chengdu, China. Eur. .J. Clin. Microbiol.. Infect. Dis., 3 3(6), 933-939. https://doi.org/10.1007/s10096-013-2029-z.

Kojima, S. \& Nikaido, H. (2013). Permeation rates of penicillins indicate that Escherichia coli porins function principally as nonspecific channels. Proc. Natl. Acad. Sci. U. S. A., 110 (28), E2629-2634. https://doi.org/10.1073/ pnas. 1310333110 .

Kosmidis, C., Schindler, B. D., Jacinto, P. L., Patel, D., Bains, K., Seo, S. M. \& Kaatz, G. W. (2012). Expression of multidrug resistance efflux pump genes in clinical and environmental isolates of Staphylococcus aureus.
Int. J. Antimicrob. Agents, 40, 204-209. https://doi. org/10.1016/j.ijantimicag.2012.04.014.

Lavigne, J. P., Sotto, A., Nicolas-Chanoine, M. H., Bouziges, N., Pagés, J. M. \& Davin-Regli, A. (2013). An adaptive response of Enterobacter aerogenes to imipenem: regulation of porin balance in clinical isolates. Int. J. Antimicrob. Agents, 41, 130-136. https://doi. org/10.1016/j.ijantimicag.2012.10.010.

Lee, C., Lee, J. H., Park, M., Park, K. S., Bae, I. K., Kim, Y, B., Cha, C., Jeong, B. C. \& Lee, S. H. (2017). Biology of Acinetobacter baumannii: Pathogenesis, antibiotic resistance mechanisms, and prospective treatment options. Front Cell Infect. Microbiol., 7, 55. https://doi. org/10.3389/fcimb.2017.00055.

Lee, H. K., Park, Y., Kim, J., Chang, E., Cho, S. G., Chae, H. S. \& Kang C. S. (2005). Prevalence of decreased susceptibility to carbapenems among Serratia marcescens, Enterobacter cloacae, and Citrobacter freundii and investigation of carbapenemases. Diagn. Microbiol. Infect. Dis., 52(4), 331-336. https://doi.org/10.1016/j. diagmicrobio.2005.04.012.

Livermore, D. M., Hope, R., Brick, G., Lillie, M. \& Reynolds, R. (2008). Non-susceptibility trends among enterobacteriaceae from bacteraemias in the UK and Ireland, 2001-2006. J. Antimicrob. Chemother, 62(2), 4154. https://doi.org/10.1093/jac/dkn351.

Lupo, A., Haenni, M. \& Madec, J. (2018). Antimicrobial resistance in Acinetobacter sp. and Pseudomonas spp. Microbiol. Spectrum., 6(3), ARBA-0007. https://doi. org/10.1128/microbiolspec.ARBA-0007-2017.

Messi, P., Guerrieri, E., Niederhaussern, S., Sabia, C. \& Bondi, M. (2006). Vancomycin-resistant enterococci (VRE) in meat and environmental samples. Int. J. Food Microbiol., 107(2), 218-222. https://doi.org/10.1016/j. ijfoodmicro.2005.08.026.

Mezzatesta, M. L., Gona, F. \& Stefani, S. (2012). Enterobacter cloacae complex: clonical impact and emerging antibiotic resistance. Future Microbiol., 7(7), 887-902. https://doi. org/10.2217/fmb.12.61.

Munita, J. M. \& Arias, C. A. (2016). Mechanism of antibiotic resistance. Microbiol. Spectr. , 4(2), VMF-0016. https:// doi.org/10.1128/microbiolspec.VMBF-0016-2015.

Nakonieczna, J., Wozniak, A., Pieranski, M., RapackaZdonczyk, A., Ogonowska, P. \& Grinholc, M. (2019). Photoinactivation of ESKAPE pathogens: overview of novel therapeutic strategy. Future Med. Chem. 11(5), 443-461. https://doi.org/10.4155/fmc-2018-0329.

Nikaido, H. (2003). Molecular basis of bacterial outer membrane permeability revisited. Microbiol. Mol. Biol. Rev., 67(4), 593-656. https://doi.org/10.1128/ mmbr.67.4.593-656.2003.

O'Neil, J. (2016). Tackling drug-resistant infections globally: final report and recommendations. Review on antimicrobial resistance. amr-review.org. https://amr- 
review.org/sites/default/files/160518_Final\%20paper_ with\%20cover.pdf. Revisado el 8 de Agosto de 2019.

Ogier, J. \& Serror, P. (2008). Safety assessment of diary microorganisms: The Enterococcus genus. Int. J. Food Microbiol., 126, 291-301. https://doi.org/10.1016/j. ijfoodmicro.2007.08.017.

Pang, Z., Raudonis, R., Glick, B. R., Lin, T. J. \& Cheng, Z. (2019). Antibiotic resistance in Pseudomonas aeruginosa: mechanisms and alternative therapeutic strategies. Biotechnol. Adv., 37 (1), 177-192. https://doi. org/10.1016/j.biotechadv.2018.11.013.

Peleg, A. Y., Seifert, H. \& Paterson, D. L. (2008). Acinetobacter baumannii: Emergence of a successful pathogen. Clin. Microbiol. Rev., 21(3), 538-582. https://doi.org/10.1128/ CMR.00058-07.

Pumbwe, L. \& Piddock, L. J. V. (2000). Two efflux systems expressed simultaneously in multidrug resistant Pseudomonas aeruginosa. Antimicrob. Agents Chemother, 44(10), 2861-2864. https://doi.org/10.1128/ aac.44.10.2861-2864.2000.

Quennan, A. M. \& Bush, K. (2007). Carbapenemases: the versatile $\beta$-Lactamases. Clin. Microbiol. Rev., 20(3), 440458. https://doi.org/10.1128/CMR.00001-07.

Rice, L. B. (2008). Federal funding for the study of antimicrobial resistance in nosocomial pathogens: No ESKAPE. J. Infect. Dis., 197(8), 1079-1081. https://doi. org/10.1086/533452.

Robicsek, A., Strahilevitz, J., Jacoby, G. A., Macielag, M., Abbanat, D., Park, C. H., Buch, K. \& Hooper, D. C. (2006). Fluoroquinolone-modifying enzyme: a new adaptation of a common aminoglycoside acethyltransferase. Nat. Med., 12(1), 83-88. https://doi.org/10.1038/nm1347.

Rossolini, G. M., D'Andrea, M. M. \& Mugnaioli, C. (2008). The spread of CTX-M-type extended-spectrum $\beta$-lactamases. Clin. Microbio. Infect., 14(1), 33-41. https://doi.org/10.1111/j.1469-0691.2007.01867.x.

Sakoulas, G. \& Moellering, R. C. (2008). Increasing antibiotic resistance among methicillin-resistant Staphylococcus aureus strains. Clin. Infect. Dis., 46(5), 360-367. https:// doi.org/10.1086/533592.

Serio, A. W., Keepers, T., Andrews, L. \& Krause, K. M. (2018). Aminoglycoside revival: review of a historically important class of antimicrobials undergoing rejuvenation. Eco. Sal. Plus, 8(1), e1-20. https://doi.org/10.1128/ ecosalplus.ESP-0002-2018.
Shore, A. C., Deasy, E. C., Slickers, P., Brenan, G., O'Connell, B., Monecke, S., Ehricht, R. \& Coleman, D. C. (2011). Detection of Staphylococcal cassette chromosome mec type XI carrying highly divergent $m e c A$, mecI, mecRI, blaZ, and ccr genes in human clinical isolates of clonal complex 130 methicillinresistant Staphylococcus aureus. Antimicrob. Agents Chemother, 55(8), 3765-3773. https://doi.org/10.1128/ AAC.00187-11.

Smith, C. A. \& Baker, E. N. (2002). Aminoglycoside antibiotic resistance by enzymatic deactivation. Curr. Drug Target sInfect. Disord., 2(2), 143-160. https://doi. org/10.2174/1568005023342533.

Sommer, M. A. O., Munck, C., Toft-Kehler, R. S. \& Andersson, D. I. (2017). Prediction of antibiotic resistance: time for a new preclinical paradigm? Nat. Rev. Microbiol., 15(11), 689-696. https://doi.org/10.1038/nrmicro.2017.75.

Tacconelli, E., Carrara, E., Savoldi, A., Harbarth. S., Mendelson, M., Monnet, D. L., Pulcini, C., Kahlmeter G., Kluytmans, J., Carmeli, Y., Ovellette, M., Outterson, K., Patel, J., Cavaleri, M., Cox, E. M., Houchens, C. R., Grayson, M. L., Hansen, P., Singh, N., Theuretzbacher, U. \& Magrini, N. (2018). Discovery, research, and the development of new antibiotics: the WHO priority list of antibiotics-resistant bacteria and tuberculosis. Lancet Infect. Dis., 18, 318-327. https://doi.org/10.1016/S14733099(17)30753-3.

Tran, Q., Williams, S., Farid, R., Erdemli, G. \& Pearlstein, R. (2013). The translocation kinetics of antibiotics through porin OmpC: insights from structure-based solvation mapping using Water Map. Proteins, 81(2), 291-299. https://doi.org/10.1002/prot.24185.

Vestergaard, M., Frees, D. \& Ingmer, H. (2019). Antibiotic resistance and the MRSA problem. Microbiol. Spectr., 7(2), GPP3-0057. https://doi.org/10.1128/microbiolspec. GPP3-0057-2018.

Viale, P., Giannella, M., Tedeschi, S. \& Lewis, R. (2015). Treatment of MDR-Gram negative infections in the 21st century: a never ending threat for clinicians. Curr. Opin. Phramacol., 24, 30-37. https://doi.org/10.1016/j. coph.2015.07.001.

Wright, P. M., Seiple, I. B. \& Myers, A. G. (2014). The evolving role of chemical synthesis in antimicrobial drug discovery. Angew. Chem. Int. Ed. Engl., 53(34), 88408869. https://doi.org/10.1002/anie.201310843. 